\title{
Radiation Induced Color Centers and Light Monitoring for Lead Tungstate Crystals ${ }^{1}$
}

\author{
Xiangdong Qu, Liyuan Zhang and Ren-yuan Zhu \\ California Institute of Technology, Pasadena, CA 91125, USA
}

\begin{abstract}
This paper presents result of a study on undoped as well as various doped lead tungstate crystals. Radiation induced color center density was compared to radio luminescence. Light output degradation for crystals under irradiation was measured. Correlations between variations of crystal transmittance and light output were investigated. Monitoring wavelength was determined so that adequate sensitivity and good linearity may be achieved.
\end{abstract}

\section{INTRODUCTION}

Because of its high density and fast decay time, lead tungstate $\left(\mathrm{PbWO}_{4}\right)$ crystal was chosen by the Compact Muon Solenoid (CMS) experiment to construct a precision electromagnetic calorimeter (ECAL) at the Large Hadronic Collider (LHC) [1]. The crystal, however, is subject to the radiation damage [2], which has been extensively studied in the last several years $[3,4,5,6,7,8,9]$. Our previous studies on $\mathrm{PbWO}_{4}$ samples concluded that although most $\mathrm{PbWO}_{4}$ crystals suffer from non-negligible radiation damage the scintillation mechanism in $\mathrm{PbWO}_{4}$ is not affected by radiation, i.e. the loss of light output is due only to absorption by radiation induced color centers [8]. Following a kinetic model [10], we also proposed that the level of the radiation damage in $\mathrm{PbWO}_{4}$ crystals should be dose rate dependent because of the damage recovery observed, which was later confirmed by experimental measurements [9].

Since the loss of transmittance, or the increase of radiation induced color center density, can be measured by a light monitoring system, we can use variations of transmittance data to estimate variations of light output. This makes possible to use a light monitoring system as an inter calibration in situ at LHC, and thus makes a precision calorimeter possible by using $\mathrm{PbWO}_{4}$ crystals which suffer from some radiation damage. In this paper, we report result of a study on undoped as well as doped $\mathrm{PbWO}_{4}$ crystals, including photo and radio luminescence spectra (Section II), radiation induced color density (Section III), light output degradation (Section IV), and correlations between variations of light output and transmittance (Section V), leading to a conclusion of choice of monitoring wavelength.

\section{Photo And Radio Luminescence}

Table 1 lists peak wavelength of Photo $\left(\lambda_{p h o}\right)$ and Radio $\left(\lambda_{\text {rad }}\right)$ luminescence for six pairs $\mathrm{PbWO}_{4}$ crystals samples of different types. All samples, except SIC 274 which has a

\footnotetext{
${ }^{1}$ Work supported in part by U.S. Department of Energy Grant No. DE-FG03-92-ER40701.
}

rectangular shape, are full size with CMS tapered geometry. Also listed in the table are date received and dopant in the crystal. Samples denoted with SIC were grown at Shanghai Institute of Ceramics, China, by modified Bridgman method. Samples denoted with BTCP were grown at Bogoroditsk Techno-Chemical Plant, Russia, by Czochralski method.

Photo luminescence was measured by using a Hitachi F-4500 fluorescence spectrophotometer. A schematic of the measurement setup is shown in Figure 1, where UV excitation light was shot to a bare surface of the sample and photo luminescence, without passing through sample, was measured by a photo multiplier tube (PMT) through a monochromator.

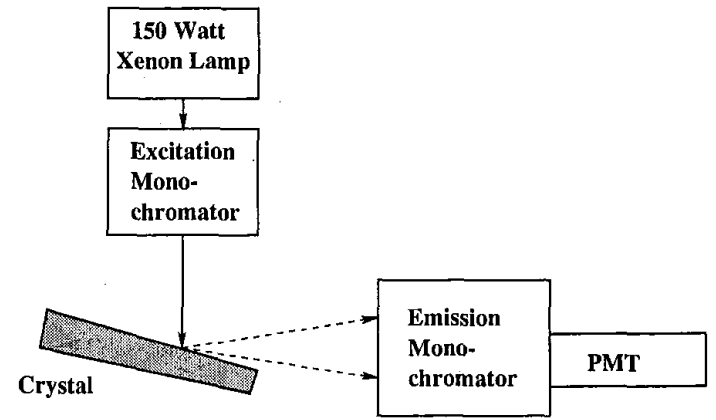

Figure 1: A schematic of setup used to measure photo luminescence.

The setup used to measure radio luminescence is shown in Figure 2, where whole body of a wrapped sample was irradiated by a ${ }^{60} \mathrm{Co} \gamma$-ray source at a dose rate of about $1,000 \mathrm{rad} / \mathrm{h}$, and the radio luminescence, passing through sample, was focused, passed through a monochromator and measured by a PMT.

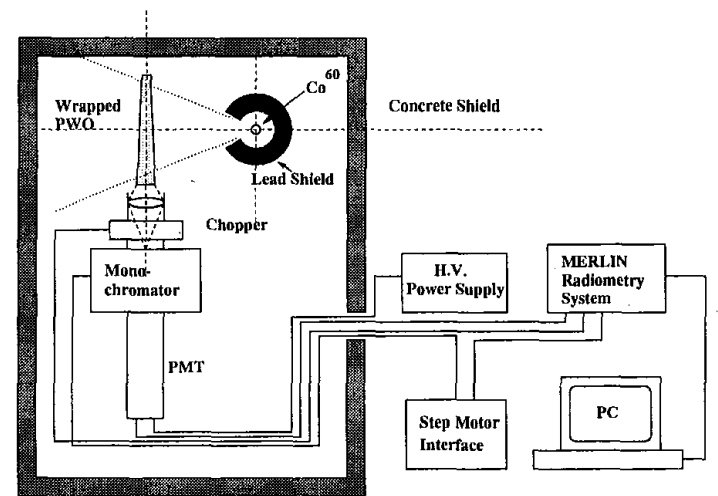

Figure 2: A schematic of setup used to measure radio luminescence.

A comparison of photo (solid) and radio (dashed) luminescence spectra is shown in Figure 3 for undoped 
Table 1

$\mathrm{PbWO}_{4}$ Crystal Samples Measured at Caltech

\begin{tabular}{cccccc}
\hline \hline ID & Dimension $(\mathrm{cm})$ & Date & Dopant & $\lambda_{\text {pho }}(\mathrm{nm})$ & $\lambda_{\text {rad }}(\mathrm{nm})$ \\
\hline SIC 167 & $2.1 \times \mathbf{2 3} \times 2.3$ & $10 / 97$ & No & 495 & 510 \\
SIC 210 & $2.1 \times \mathbf{2 3} \times 2.3$ & $5 / 98$ & No & 495 & 510 \\
\hline SIC 274 & $2.5 \times \mathbf{2 1} \times 2.5$ & $10 / 98$ & Sb & 510 & 450 \\
SIC 275 & $2.2 \times \mathbf{2 3} \times 2.6$ & $10 / 98$ & Sb & 510 & 450 \\
\hline BTCP-1965 & $2.1 \times \mathbf{2 3} \times 2.3$ & $10 / 97$ & La & 420 & 435 \\
BTCP-1971 & $2.1 \times \mathbf{2 3} \times 2.3$ & $5 / 98$ & La & 420 & 435 \\
\hline BTCP-2133 & $2.2 \times \mathbf{2 3} \times 2.5$ & $4 / 99$ & Y/Nb & 420 & 435 \\
BTCP-2162 & $2.2 \times \mathbf{2 3} \times 2.5$ & $4 / 99$ & Y/Nb & 420 & 435 \\
\hline SIC S301 & $2.2 \times \mathbf{2 3} \times 2.6$ & $6 / 99$ & Y/Sb & 420 & 445 \\
SIC S347 & $2.2 \times \mathbf{2 3} \times 2.6$ & $6 / 99$ & Y/Sb & 420 & 445 \\
\hline SIC S392 & $2.2 \times \mathbf{2 3} \times 2.6$ & $6 / 99$ & Y & 420 & 445 \\
SIC S412 & $2.2 \times \mathbf{2 3} \times 2.6$ & $6 / 99$ & Y & 420 & 445 \\
\hline \hline
\end{tabular}

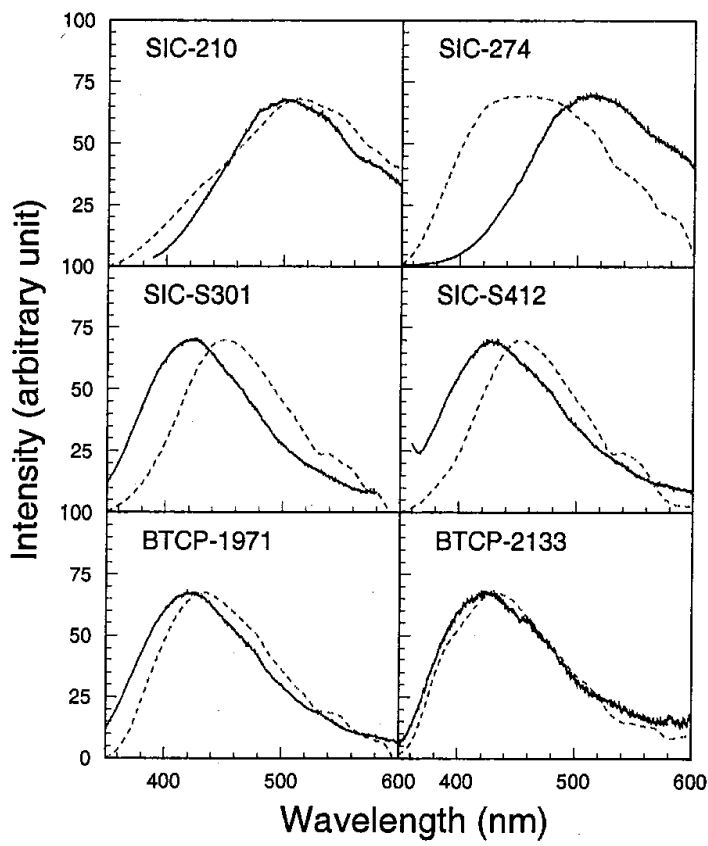

Figure 3: A comparison of photo (solid) and radio (dashed) luminescence spectra for six $\mathrm{PbWO}_{4}$ samples.

(SIC-210), Sb doped (SIC-274), Y/Sb double doped (SIC-S301), Y doped (SIC-S412), La doped (BTCP-1971) and $\mathrm{Y} / \mathrm{Nb}$ double doped (BTCP-2133) samples. All spectra were corrected by monochromator efficiency and PMT quantum efficiency. The vertical axis "Intensity" refers to photon numbers, and its scale is arbitrary. As seen from the figure the peak of radio luminescence is 15 to $25 \mathrm{~nm}$ red shifted as compared to the photo luminescence for all samples, except $\mathrm{Sb}$ doped sample SIC-274. This red shift can be explained by internal absorption since $\mathrm{PbWO}_{4}$ transmittance at shorter wavelength is poorer than that at longer wavelength. A cross check of photo luminescence with a modified setup, where luminescence light passed through sample, showed similar red shift, and thus confirmed this explanation.

The $\mathrm{Sb}$ doped samples are significantly different, however. As shown in Figure 3, the peak of radio luminescence (dashed) is blue shifted by $60 \mathrm{~nm}$ as compared to the photo luminescence (solid). The origin of this blue shift is suspected due to that the UV photon is not energetic enough to excite the scintillation centers in Sb doped sample. This was confirmed by an Xray luminescence spectrum with a peak consistent with radio luminescence.

Since the photo luminescence spectrum measured with setup in Figure 1 is not affected by internal absorption, it can be seen as the intrinsic emission spectrum in general, while the radio luminescence spectrum is a convolution of intrinsic emission and internal absorption with later depends on the light path. For $\mathrm{PbWO}_{4}$ monitoring, however, the photo detector quantum efficiency weighted radio luminescence spectrum is more relevant since scintillation light propagates through the crystal then reaches the photo detector. Measurement on the other sample of the same type in Table 1 provided consistent result.

\section{RAdiATION INDUCED COLOR CENTERS}

It is known that color centers are created in $\mathrm{PbWO}_{4}$ crystals under irradiation. It is also known that radiation induced color centers may annihilate under room temperature. During irradiation, both annihilation and creation coexist, the color center density will reach an equilibrium at a level depending on the dose rate applied. Assuming the annihilation speed of color center $i$ is proportional to a constant $a_{i}$ and its creation speed is proportional to a constant $b_{i}$ and the dose rate $(R)$, the differential variation of color center density when both processes coexist can be written as [10]

$$
d D=\sum_{i=1}^{n}\left\{-a_{i} D_{i}+\left(D_{i}^{\text {all }}-D_{i}\right) b_{i} R\right\} d t
$$


where $D_{i}$ is the density of the color center $i$ in the crystal and the summation goes through all centers. The solution of Equation 1 is

$$
D=\sum_{i=1}^{n}\left\{\frac{b_{i} R D_{i}^{a l l}}{a_{i}+b_{i} R}\left[1-e^{-\left(a_{i}+b_{i} R\right) t}\right]+D_{i}^{0} e^{-\left(a_{i}+b_{i} R\right) t}\right\}
$$

where $D_{i}^{\text {all }}$ is the total density of the trap related to the center $i$ and $D_{i}^{0}$ is its initial density.

The color center density in equilibrium $\left(D_{e q}\right)$ thus depends on the dose rate $(R)$ :

$$
D_{e q}=\sum_{i=1}^{n} \frac{b_{i} R D_{i}^{a l l}}{a_{i}+b_{i} R}
$$

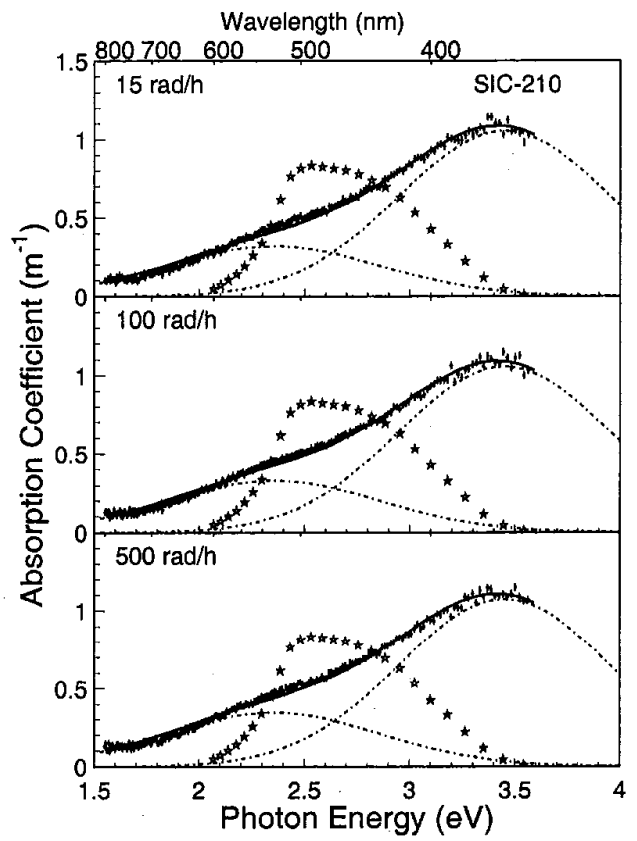

Figure 4: Radiation induced color center density for a undoped sample SIC-210

Figures 4, 5, 6, 7, 8 and 9 show radiation induced color center density as function of photon energy for undoped (SIC-210), Sb doped (SIC-275), La doped (BTCP-1971), Y/Nb double doped (BTCP-2133), Y/Sb double doped (SIC-S301) and Y doped (SIC-S392) samples, respectively, in equilibrium at different dose rates. The stars in these figures represent corresponding radio luminescence spectra weighted with quantum efficiency of the PMT used in monitoring test bench, which is described in Section V. The points with error bars in these figures are radiation induced color center density (D), or absorption coefficient, measured in equilibrium under dose rates specified. It was calculated according to equation

$$
D=1 / L A L_{\text {equilibrium }}-1 / L A L_{\text {before }}
$$

where LAL is light attenuation length calculated by using longitudinal transmittance according to Equation 1 of

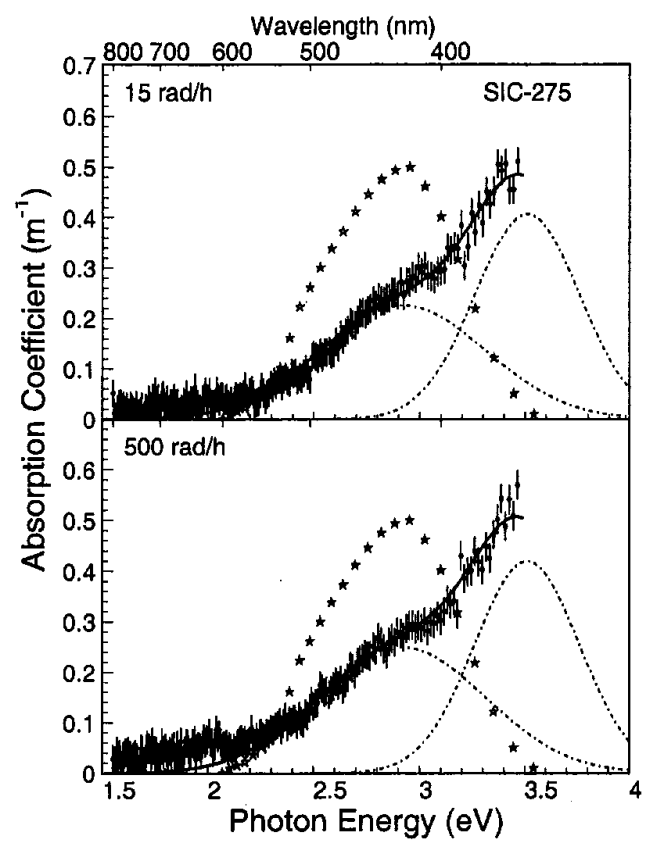

Figure 5: Radiation induced color center density for a $\mathrm{Sb}$ doped sample SIC-275.

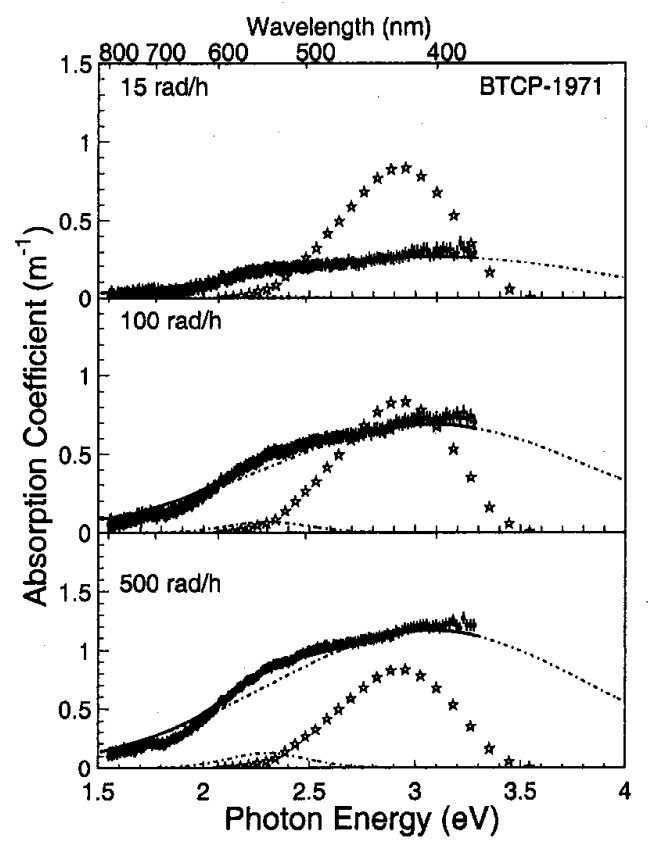

Figure 6: Radiation induced color center density for a La doped sample BTCP-1971.

reference [11], and the subscript "equilibrium" and "before" refer to "in equilibrium" and "before irradiation" respectively. The radiation induced color center density was decomposed to a sum (solid line) of two color centers:

$$
D=\sum_{i=1}^{2} A_{i} e^{-\frac{\left(E-E_{i}\right)^{2}}{2 \sigma_{i}^{2}}}
$$




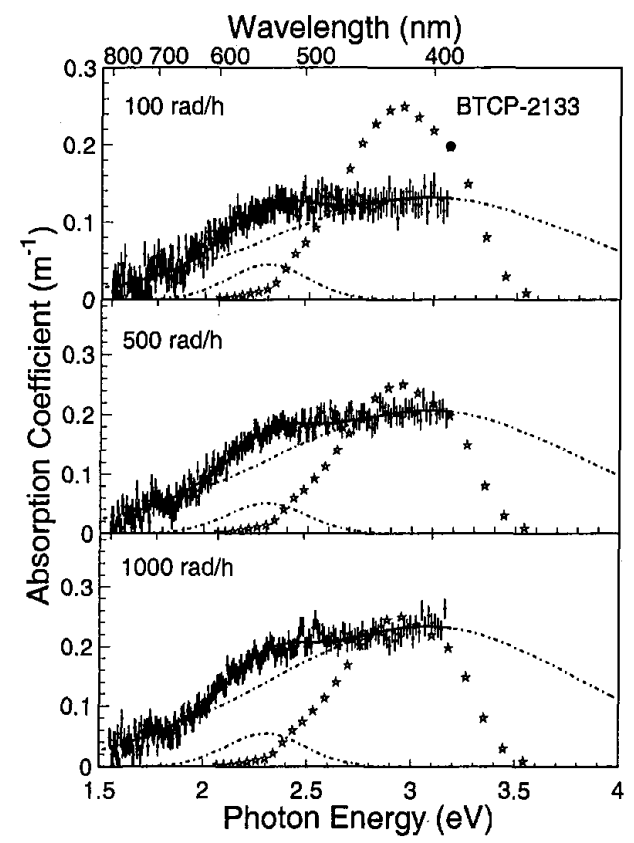

Figure 7: Radiation induced color center density for a $\mathrm{Y} / \mathrm{Nb}$ doped sample B'TCP-2133.

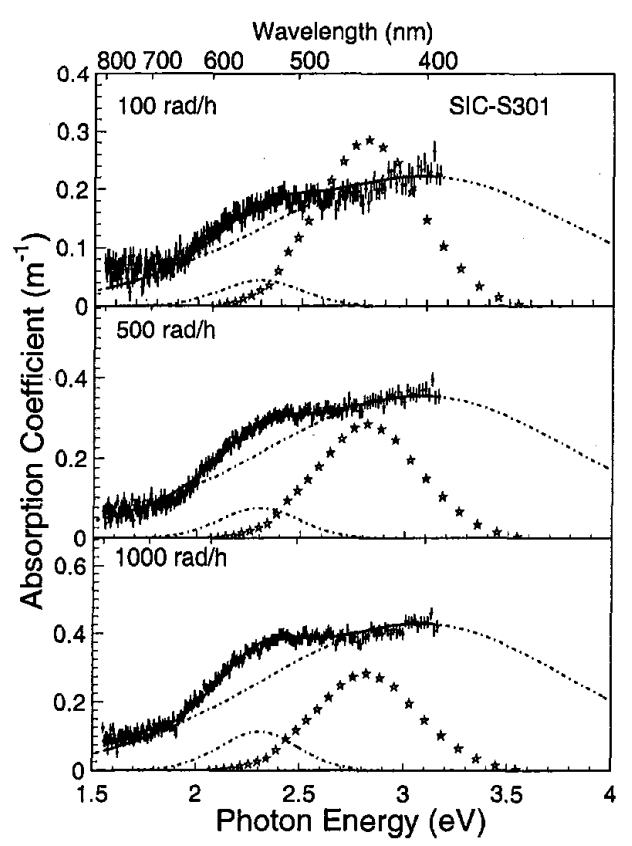

Figure 8: Radiation induced color center density for a $\mathrm{Y} / \mathrm{Sb}$ doped sample SIC-S301.

where $E_{i}, \sigma_{i}$ and $A_{i}$ denote the energy, width and amplitude of the color center $i$, and $E$ is photon energy. As seen from these figures, the fit with two centers of Gaussian shape (dashed lines) provides a rather good description of the radiation induced color center data with $\chi^{2} / \mathrm{DoF}$ less than one.

Table 2 lists numerical result of the fit for all 12 samples listed in Table 1, where S and B represent SIC and BTCP

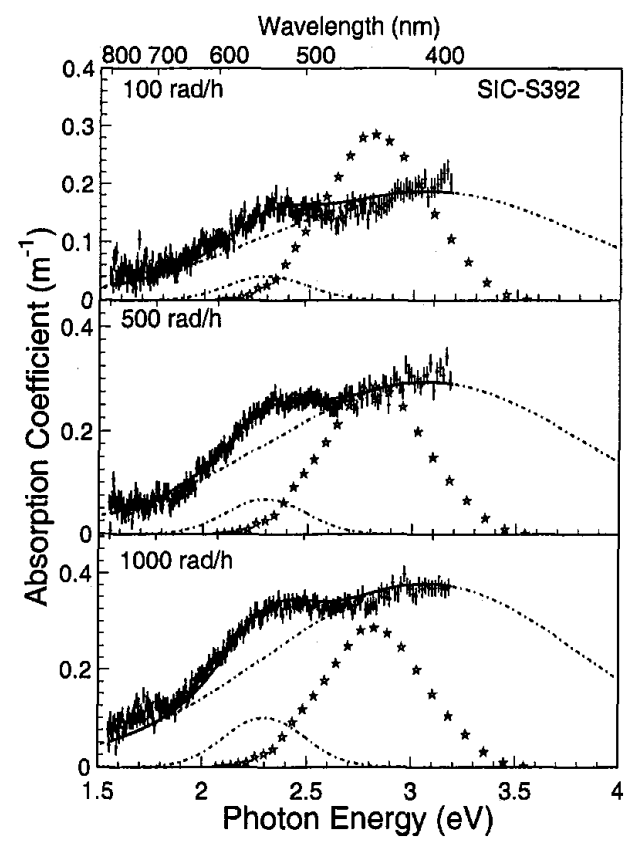

Figure 9: Radiation induced color center density for a $Y$ doped sample SIC-S392.

respectively. Consistent fit result was found for the same type of two samples. It is interesting to note that all tri-valent (La, $\mathrm{Y} / \mathrm{Nb}, \mathrm{Y} / \mathrm{Sb}$ and $\mathrm{Y}$ ) doped samples have two common radiation induced color centers at the same energy and with the same width. One broad center is at wavelength of $400 \mathrm{~nm}(3.07 \mathrm{eV})$ with a width of $0.76 \mathrm{eV}$, and other narrow center is at a longer wavelength of $540 \mathrm{~nm}(2.30 \mathrm{eV})$ with a width of $0.19 \mathrm{eV}$. The radiation induced color centers in undoped and $\mathrm{Sb}$ doped samples, however, are rather different.

It is also interesting to note that these two color centers are peaked well apart at two sides of the peak of radio luminescence, and they have different damage and annihilation constants. This indicates that $\mathrm{PbWO}_{4}$ monitoring light wavelength must be carefully chosen so that variations of crystal light output may be adequately estimated by using variations of transmittance.

\section{LIGHT OUTPUT DEGRADATION UNDER RADIATION}

Spectroscopically speaking, crystal light output is a convolution of radio luminescence, transmittance and quantum efficiency of photo detector. Following Equation 3, $\mathrm{PbWO}_{4}$ light output degradation under irradiation is also dose rate dependent since luminescence and photo detector quantum efficiency are not affected by radiation, or at least not in the same time scale. Figure 10 shows light output normalized to before irradiation (solid dots with error bars) as function of time for undoped (SIC-167), Sb doped (SIC-274), La doped (BTCP-1965), Y/Nb double doped (BTCP-2133), Y/Sb double doped (SIC-S301) and Y doped (SIC-S412) samples under lateral irradiation by a ${ }^{60} \mathrm{Co} \gamma$-ray source. Note, lateral irradiation provides a uniform dose profile of entire body of the 
Table 2

Summary of Radiation Induced Color Centers

\begin{tabular}{ccccccccccc}
\hline \hline ID & $\begin{array}{c}\mathrm{E}_{1} / \sigma_{1} \\
\mathrm{eV} / \mathrm{eV}\end{array}$ & $\begin{array}{c}\mathrm{A}_{1}^{a} \\
\mathrm{~m}^{-1}\end{array}$ & $\begin{array}{c}\mathrm{A}_{1}^{b} \\
\mathrm{~m}^{-1}\end{array}$ & $\begin{array}{c}\mathrm{A}_{1}^{c} \\
\mathrm{~m}^{-1}\end{array}$ & $\begin{array}{c}\mathrm{A}_{1}^{d} \\
\mathrm{~m}^{-1}\end{array}$ & $\begin{array}{c}\mathrm{E}_{2} / \sigma_{2} \\
\mathrm{eV} / \mathrm{eV}\end{array}$ & $\begin{array}{c}\mathrm{A}_{2}^{a} \\
\mathrm{~m}^{-1}\end{array}$ & $\begin{array}{c}\mathrm{A}_{2}^{b} \\
\mathrm{~m}^{-1}\end{array}$ & $\begin{array}{c}\mathrm{A}_{2}^{c} \\
\mathrm{~m}^{-1}\end{array}$ & $\begin{array}{c}\mathrm{A}_{2}^{d} \\
\mathrm{~m}^{-1}\end{array}$ \\
\hline $\mathrm{S}-167$ & $2.35 / 0.51$ & 0.23 & 0.39 & 0.47 & - & $3.45 / 0.50$ & 0.99 & 0.88 & 1.07 \\
$\mathrm{~S}-210$ & $2.35 / 0.51$ & 0.20 & 0.21 & 0.22 & - & $3.45 / 0.50$ & 0.91 & 0.91 & 0.93 & - \\
\hline $\mathrm{S}-274$ & $2.92 / 0.39$ & 0.33 & - & 0.38 & - & $3.51 / 0.25$ & 0.88 & - & 0.90 & - \\
$\mathrm{S}-275$ & $2.92 / 0.39$ & 0.22 & - & 0.24 & - & $3.51 / 0.25$ & 0.40 & - & 0.41 & - \\
\hline $\mathrm{B}-1965$ & $2.30 / 0.19$ & 0.00 & 0.04 & 0.05 & - & $3.07 / 0.76$ & 0.10 & 0.22 & 0.46 & - \\
$\mathrm{B}-1971$ & $2.30 / 0.19$ & 0.01 & 0.06 & 0.12 & - & $3.07 / 0.76$ & 0.26 & 0.69 & 1.16 & - \\
\hline $\mathrm{B}-2133$ & $2.30 / 0.19$ & 0.00 & 0.04 & 0.05 & 0.05 & $3.07 / 0.76$ & 0.10 & 0.13 & 0.20 & 0.23 \\
$\mathrm{~B}-2162$ & $2.30 / 0.19$ & 0.01 & 0.03 & 0.06 & 0.06 & $3.07 / 0.76$ & 0.10 & 0.19 & 0.30 & 0.30 \\
\hline $\mathrm{S}-\mathrm{S} 301$ & $2.30 / 0.19$ & 0.00 & 0.04 & 0.07 & 0.11 & $3.07 / 0.76$ & 0.10 & 0.22 & 0.35 & 0.42 \\
$\mathrm{~S}-\mathrm{S} 347$ & $2.30 / 0.19$ & 0.00 & 0.00 & 0.03 & 0.07 & $3.07 / 0.76$ & 0.10 & 0.13 & 0.14 & 0.38 \\
\hline $\mathrm{S}-\mathrm{S} 392$ & $2.30 / 0.19$ & 0.00 & 0.04 & 0.06 & 0.10 & $3.07 / 0.76$ & 0.10 & 0.18 & 0.29 & 0.37 \\
$\mathrm{~S}-\mathrm{S} 412$ & $2.30 / 0.19$ & 0.00 & 0.03 & 0.04 & 0.06 & $3.07 / 0.76$ & 0.10 & 0.15 & 0.19 & 0.24 \\
\hline \hline
\end{tabular}

a,b,c,d represent $15,100,500,1000 \mathrm{rad} / \mathrm{h}$ respectively.

sample, so provides a more severe radiation environment as compared to front irradiation [9]. Also shown in the figure is the dose rate applied. Once again, the dose rate dependence of light output is clearly observed.

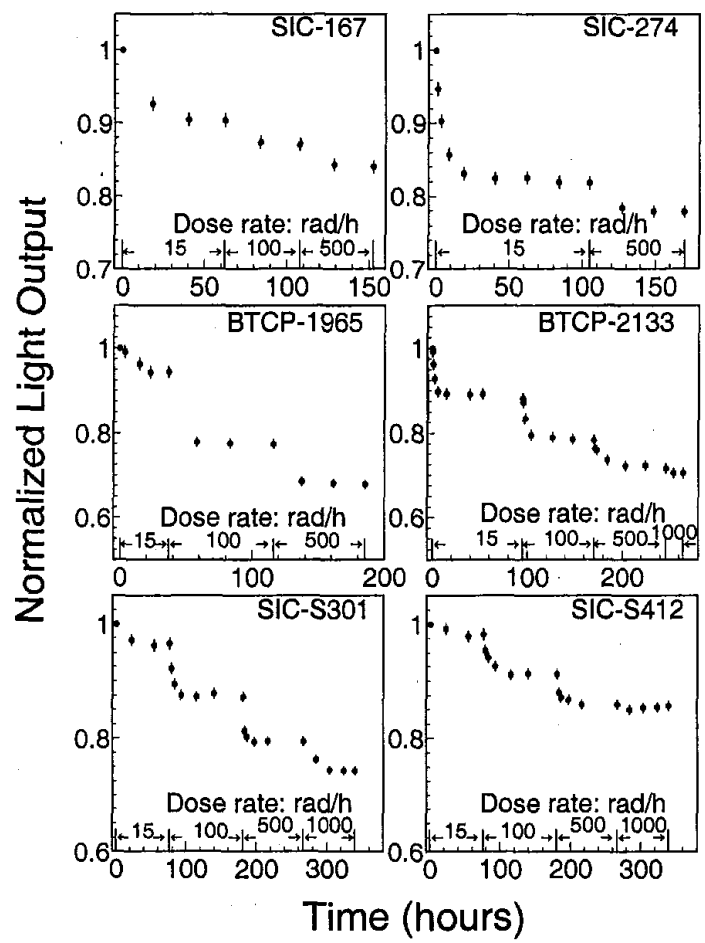

Figure 10: Normalized light output is shown as function of time after irradiation for six $\mathrm{PbWO}_{4}$ crystals.

Table 3 lists the normalized light output, in percentage, in equilibrium under different dose rate for all 12 samples, where $\mathrm{R}, \mathrm{S}$ and $\mathrm{B}$ represent Dose Rate, SIC and BTCP respectively.

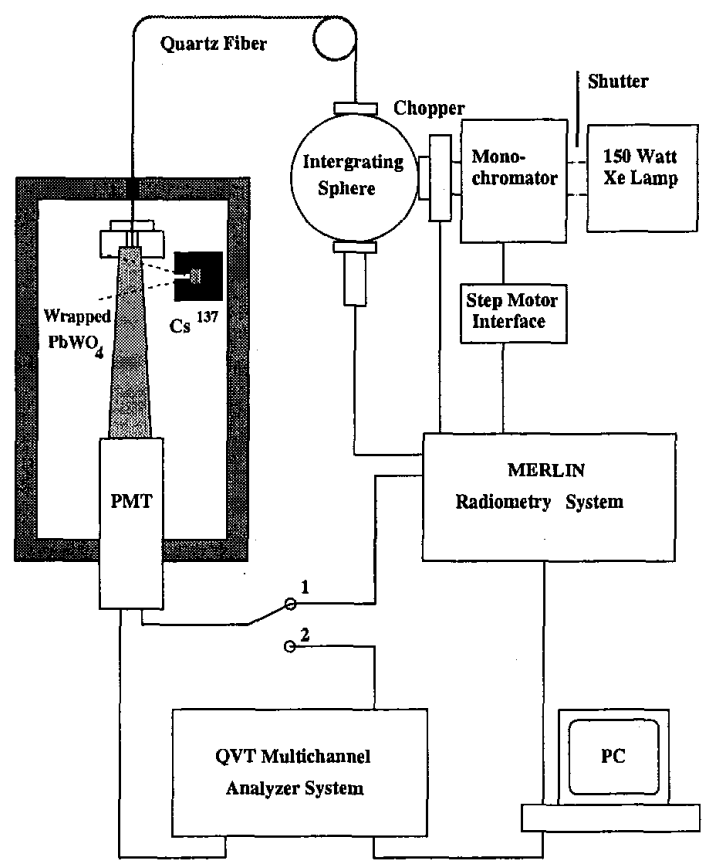

Figure 11: The monitoring test bench for light monitoring (position 1) and light output measurement (position 2).

\section{Lead Tungstate Crystal Monitoring}

As discussed in Section $\mathrm{I}$, the crucial inter calibration in situ at $\mathrm{LHC}$ for $\mathrm{CMS} \mathrm{PbWO}_{4}$ ECAL may be provided by a light monitoring system [2], which uses relative variations of transmittance to estimate relative variations of light output for each individual crystal. Figure 11 is a schematic showing a monitoring test bench at Caltech. Monitoring light from a Xenon lamp source went through a monochromator and was injected to an integrating sphere. The light from one output of the sphere was coupled to the front end of the sample through a quartz fiber and an air gap. The light from other 
Table 3

Summary of Light Output Degradation (\%) in Equilibrium under Irradiation

\begin{tabular}{ccccccccccccc}
\hline $\mathrm{R}$ & $\mathrm{S}$ & $\mathrm{S}$ & $\mathrm{S}$ & $\mathrm{S}$ & $\mathrm{B}$ & $\mathrm{B}$ & $\mathrm{B}$ & $\mathrm{B}$ & $\mathrm{S}$ & $\mathrm{S}$ & $\mathrm{S}$ & $\mathrm{S}$ \\
$\mathrm{rad} / \mathrm{h}$ & 167 & 210 & 274 & 275 & 1965 & 1971 & 2133 & 2162 & $\mathrm{~S} 301$ & $\mathrm{~S} 347$ & $\mathrm{~S} 392$ & $\mathrm{~S} 412$ \\
\hline 15 & 90.4 & 80.0 & 82.0 & 83.6 & 94.5 & 72.2 & 89.2 & 86.1 & 96.6 & 95.1 & 98.2 & 98.2 \\
100 & 87.1 & 77.0 & & & 77.9 & 47.2 & 78.6 & 76.8 & 87.3 & 88.6 & 91.3 & 91.2 \\
500 & 84.0 & - & 78.0 & \multirow{2}{*}{80.0} & 83.6 & - & 72.3 & 70.3 & 79.5 & 82.1 & 78.0 & 85.9 \\
1,000 & & & & & & & 70.5 & 68.2 & 74.3 & 78.0 & 80.2 & 85.3 \\
\hline \hline
\end{tabular}

output of the sphere was measured by a photo detector as a reference. The sample was wrapped with Tyvek paper and optically coupled to a PMT. The output of the PMT is coupled to either a Merlin from ORIEL through a lock-in amplifier for transmittance measurement (position 1), or a LeCroy QVT multichannel analyzer for light output measurement (position 2). When the switch is in position 2, the shutter at the input of monochromator is closed so that there is no interference between scintillation light and monitoring light.

The transmittance as a function of wavelength was measured in position 1 by using normalizing PMT output to that of the reference detector to reduce systematic uncertainties caused by fluctuations of the intensity of the light source. Figure 12 shows a distribution of PMT readout normalized to the reference detector on sphere, taken in 23 hours. A Gaussian fit is also shown in the figure together with the fit result of average $(\mu)$ and width $(\sigma)$. The stability, defined as $\sigma / \mu$ of the fit, was found to be better than $0.1 \%$.

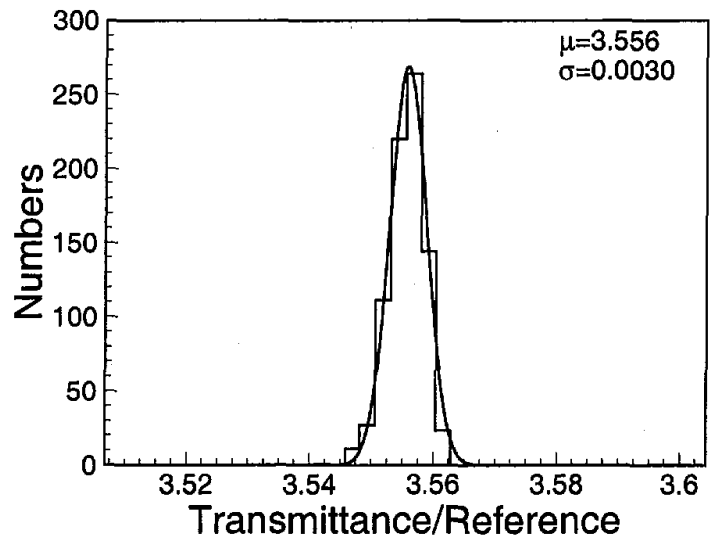

Figure 12: Distribution of PMT readout (position 1) normalized to reference detector on sphere, taken in 23 hours is shown with a Gaussian fit.

For absolute light output measurement the sample was shot by a small ${ }^{137} \mathrm{Cs}$ source. To simulate radiation environment in situ at LHC, the sample was either irradiated by a ${ }^{60} \mathrm{Co}$ source under 15 to $1,000 \mathrm{rad} / \mathrm{h}$ or under recovery after irradiation. This setup simulates what to be used in situ at LHC except that the readout detector is a PMT which covers entire back face of the sample, while photo detector used in CMS ECAL is Si avalanche photo diode (APD), which covers only a small
Normalized Light Output (\%)



Figure 13: Correlations between relative variations of transmittance and light output are shown for four monitoring wavelengths for a $\mathrm{Y} / \mathrm{Sb}$ double doped sample SIC-S301.

fraction of sample back face.

Figure 13 shows typical correlations between relative variations of transmittance $(\Delta \mathrm{T} / \mathrm{T})$ and light output $(\Delta \mathrm{LY} / \mathrm{LY})$ for monitoring light at four different wavelengths: 400,450 , 500 and $550 \mathrm{~nm}$ for a Y/Sb double doped sample SIC-S301. The correlations were fit to a linear function:

$$
\frac{\Delta \mathrm{T}}{\mathrm{T}}=\text { slope } \times \frac{\Delta \mathrm{LY}}{\mathrm{LY}}
$$

The result of the fit, the $\chi^{2} / \mathrm{DoF}$ and the slope, is also listed in the figure. While the slope represents the sensitivity for $\mathrm{PbWO}_{4}$ monitoring, the $\chi^{2} / \mathrm{DoF}$ represents the linearity of the fit. From the figure it is clear that the linearity is generally good when light output loss is less than $15 \%$, and some systematic deviations exist for monitoring light of 400,500 and $550 \mathrm{~nm}$, as compared to $450 \mathrm{~nm}$, since not all wavelengths are adequate for the monitoring. Similar correlations were observed for other samples.

Figure 14 shows monitoring sensitivity (solid dots, left scale), or slope, defined as $\frac{\Delta T}{T} / \frac{\Delta L Y}{L Y}$, and linearity (open dots, 


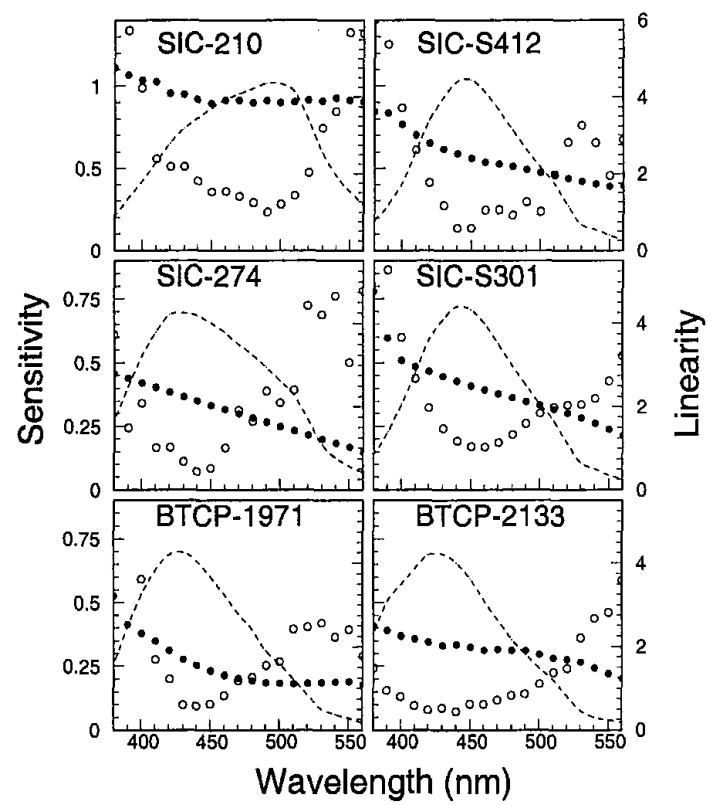

Figure 14: Monitoring sensitivity and linearity are shown as function of wavelength for six $\mathrm{PbWO}_{4}$ samples.

right scale), defined as $\chi^{2} / \mathrm{DoF}$, as function of monitoring wavelength for undoped (SIC-210), Y doped (SIC-S412), Sb doped (SIC-274), Y/Sb double doped (SIC-S301), La doped (BTCP-1971) and Y/Nb double doped (BTCP-2133) $\mathrm{PbWO}_{4}$ samples. Also shown in the figure is the corresponding PMT quantum efficiency weighted radio luminescence (dashed lines). While a shorter monitoring wavelength is preferred for a better sensitivity, the best linearity is achieved at the wavelength which coincides with the peak of radio luminescence. The better monitoring sensitivity at shorter wavelength is understood because of the poorer initial transmittance as compared to that at longer wavelength. The best linearity around the peak of radio luminescence is understood by two radiation induced color centers discussed in Section III.

\section{SUMMARY}

We measured photo and radio luminescence, radiation induced color centers, light output degradation and monitoring sensitivity and linearity for six types of $\mathrm{PbWO}_{4}$ crystals: undoped, La doped, Sb doped, Y/Nb double doped, Y/Sb double doped, and $\mathrm{Y}$ doped. All tri-valent doped samples, including $\mathrm{La}, \mathrm{Y} / \mathrm{Nb}, \mathrm{Y} / \mathrm{Sb}$ and $\mathrm{Y}$ doped samples, have consistent intrinsic photo luminescence peaked at $420 \mathrm{~nm}$. Because of internal absorption, their radio luminescence is 15 to $25 \mathrm{~nm}$ red shifted. Similarly, the peak of radio luminescence of undoped $\mathrm{PbWO}_{4}$ crystals is $15 \mathrm{~nm}$ red shifted from the photo luminescence ( $495 \mathrm{~nm}$ ). The only exception is $\mathrm{Sb}$ doped $\mathrm{PbWO}_{4}$. Its peak of radio luminescence is $60 \mathrm{~nm}$ blue shifted from photo luminescence $(510 \mathrm{~nm})$, explained by not energetic enough photons of the UV excitation light.

Radiation induced color center density in all samples can be decomposed to two color centers. All tri-valent doped samples have two common radiation induced color centers peaked at 400 and $540 \mathrm{~nm}$. The peaks of radiation induced color centers for undoped samples are at 360 and $530 \mathrm{~nm}$, and that of $\mathrm{Sb}$ doped samples are at 350 and $430 \mathrm{~nm}$. These two centers are located at two sides of luminescence and with different damage and recovery constant. The physical nature of these color centers are not yet identified.

Because it is less transparent at short wavelengths, the monitoring sensitivity for $\mathrm{PbWO}_{4}$ crystal is better at short wavelength. The best linearity, however, can only be achieved with monitoring light wavelength around the peak of radio luminescence weighted with quantum efficiency of photo detector. Since CMS ECAL uses readout device of APD which has a flat quantum efficiency as compared to that of the PMT with bi-alkali photo cathode used in this investigation, it is expected that the monitoring wavelength of choice is around $440 \mathrm{~nm}$ for all tri-valent or $\mathrm{Sb}$ doped $\mathrm{PbWO}_{4}$ crystals. The undoped crystal, however, would require a monitoring wavelength around $510 \mathrm{~nm}$.

\section{ACKNOWLEDGEMENTS}

Prof. Z.W. Yin and P. Lecoq provided samples in this report. Dr. Q. Deng measured sample SIC-167 and BTCP-1965 when he was at Caltech. Many interesting discussions with Drs. F. Nessi, P. Lecomte, J.Y. Liao, D.Z. Shen, D.S. Yan and Z.W. Yin are acknowledged.

\section{REFERENCES}

[1] Compact Muon Solenoid Technical Proposal, CERN/LHCC 94-38, LHCC/P1 (1994).

[2] CMS Collaboration, The Electromagnetic Calorimeter Technical Design Report, CERN/LHCC 97-33 (1997).

[3] H.F. Chen fet al., Nucl. Instr. and Meth. A414 (1998) 149.

[4] P. Lecoq et al., Nucl. Instr. and Meth. A403 (1998) 302, Nucl. Instr. and Meth. A402 (1998) 75, and Nucl. Instr. and Meth. A365 (1995) 291.

[5] M. Kobayashi et al., Nucl. Instr. and Meth. A406 (1998) 442, Nucl. Instr. and Meth. A404 (1998) 149, Nucl. Instr. and Meth. A399 (1997) 261, and Nucl. Instr. and Meth. A373 (1996) 333.

[6] M. Korzhik et al., Rad. Meas. 29(1) (1998) 27, J. Allo. \& Comp, 238 (1996) 46, Phys. Stat. Sol. a156 (1996) 493, and Phys. Stat. Sol. a154 (1996) 779.

[7] M. Nikl et al., Phys. Stat. Sol. a164 (1997) R9, J. Appl. Phys. Lett. 82(11) (1997) 5758, J. Appl. Phys. Lett. 71(26) (1997) 3755, Phys. Stat. Sol. b196 (1996) K7, and Phys. Stat. Sol. b195 (1996) 311.

[8] C. Woody et al., IEEE Trans. Nucl. Sci. NS-43 (1996) 1585, and Nucl. Instr. and Meth. A376 (1996) 319.

[9] R.Y. Zhu et al., Nucl. Instr. and Meth. A413 (1998) 297, IEEE Trans. Nucl. Sci. NS-45 (1998) 686, and IEEE Trans. Nucl. Sci. NS-44 (1997) 468.

[10] D. Ma and R.Y. Zhu, Nucl. Instr. and Meth. A332 113 (1993).

[11] R.Y. Zhu et al., Nucl. Instr. and Meth. A376 (1996) 319. 